\title{
Input Constrained Positioning Control for a Class of Euler-Lagrange Systems with Discontinuities
}

\author{
Kai Zheng Non-member (Harbin Institute of Technology, hitkzh@gmail.com) \\ Tielong Shen Member (Sophia University, tetu-sin@sophia.ac.jp) \\ Yu Yao Non-member (Harbin Institute of Technology, yaoyu@hit.edu.cn)
}

Keywords: positioning control, Euler-Lagrange systems, nonsmooth control

In high accuracy position servo systems, how to overcome the drawbacks caused by static friction, such as stick-slip limit-cycling, large steady-state errors, are still a challenging problem in practice. Meanwhile, due to the contraints of acturator, some high gain control schemes are not feasible for implementation.

In order to coordinate the accuracy requirement and the capacity of actuator, this paper proposes a nonlinear positioning control scheme for a class of mechanical systems which are modeled by Euler-Lagrange equation. Due to nonsmooth static friction, the error dynamics of the closed-loop system with any static Lipschitz feedback controller is a typical differential equation with discontinuous right-hand side. For such a nonsmooth system, the differential inclusion theory given by Filippov is introduced to analyze the solution at first, i.e. all solutions of the closed loop are determined by the following differential inclusion

$$
\begin{aligned}
\dot{x} & \in K[f](x) \\
& =K\left[\begin{array}{c}
x_{2} \\
-G(q) C(q, \dot{q}) x_{2}+G(q) \operatorname{Sat}\left(\tau_{r}\right)-G(q) D \operatorname{Sgn}\left(x_{2}\right)
\end{array}\right]
\end{aligned}
$$

The first result, in the paper, shows that the static sulotion of the closed-loop system is not unique but a set related to the control law.

Lemma 1. For the closed-loop system with any static Lipschitz feedback controller $\tau_{r}\left(x_{1}, x_{2}\right)$ which satisfies $\tau_{r}=\operatorname{Sat}\left(\tau_{r}\right)$, i.e. $\left|\tau_{r_{i}}\right| \leq B_{i}, i=1, \ldots, n$, any $x \in \Omega$ is an equilibrium point of the closed-loop system, where

$$
\Omega=\left\{x \in R^{2 n} \mid \tau_{r_{i}}\left(x_{1}, 0\right) \in d_{i} K[\operatorname{sgn}(0)], x_{2}=0, i=1, \cdots, n\right\}
$$

Lemma 1 also indicates that the static solution may be bounded. With the hint from Lemma 1 , a $P D$-like feedback controller is proposed, that is

$$
\tau_{r}=-K_{p} \operatorname{Arctg}\left(H x_{1}\right)-K_{d} \operatorname{Arctg}\left(x_{2}\right)
$$

where the arctangent function is introduced to filter the feedback signals.

Moreover, due to the boundness of the arctangent function, the controller satisfies the condition of Lemma 1. As the second result provided in this paper, the following theorem shows that with the $P D$-like controller, the static solution set has a similar form as the set given in Lemma 1.

Theorem 1. For the closed-loop system with the $P D$-like controller proposed, if the design parameters and $\boldsymbol{K}_{p}, \boldsymbol{K}_{\boldsymbol{d}}$ satisfy

$$
k_{p_{i}}+k_{d_{i}} \leq 2 \pi B_{i}, i=1, \cdots, n,
$$

then any $x=\left[\begin{array}{ll}x_{1}^{T} & x_{2}^{T}\end{array}\right]^{T} \in \Omega$ is an equilibrium of the closed-loop system, where

$$
\Omega=\left\{x \in R^{2 n}|| x_{1_{j}} \mid \leq \frac{1}{h_{j}} \tan \left(\frac{d_{j}}{k_{p_{j}}}\right), x_{2}=0, j=1, \cdots, n\right\}
$$

From Theorem 1, it's obvious that the range of static error is related to the design parameter $\boldsymbol{H}$ and $\boldsymbol{K}_{p}$. By taking advantage of a non-quadratic Lyapunov function candidate, the stability of the closed-loop system can be obtained, which is the third result of this paper shown as Theorem 2 .

Theorem 2. Consider the system with the $P D$-like controller. For any prespecified positive value $\varepsilon_{i}, i=1, \ldots, n$ and any initial condition, if $\boldsymbol{H}, \boldsymbol{K}_{p}, \boldsymbol{K}_{\boldsymbol{d}}$ satisfy

$$
\begin{aligned}
& \text { 1. } k_{p_{i}}+k_{d_{i}} \leq 2 \pi B_{i}, i=1, \cdots, n ; \\
& \text { 2. } k_{p_{i}} \leq 2 d_{i}, i=1, \cdots, n ; \\
& \text { 3. } \frac{1}{h_{i}} \tan \left(\frac{d_{i}}{k_{p_{i}}}\right)<\varepsilon_{i}, i=1, \cdots, n,
\end{aligned}
$$

then the closed loop is globally stable and $\lim _{t \rightarrow \infty}\left|x_{i}\right|=c_{i} \leq \varepsilon_{i}$, $i=1, \ldots, n$.

Three conditions in Theorem 2 help us to choose the feasible design parameters. Condition 1 guarantees that the controller works in the linear region of the actuator. For condition 2, it aims for the stability of the closed-loop system. Condition 3 is introduced for the accuracy requirement. It's clear that the system stability and positioning accuarcy are guaranteed by different design parameters. This is another advantage of this design.

Finally, a numerical example is carried out to illustrate the effect of the proposed $P D$-like controller. A two degree of freedom planner manipulator is examined as the plant. The results show that the driving torque is in the linear region of the saturation although the initial state has large variation. And the servo accuracy is also maintained. 


\title{
Input Constrained Positioning Control for a Class of Euler-Lagrange Systems with Discontinuities
}

\author{
Kai Zheng* Non-member \\ Tielong Shen ${ }^{* *}$ Member \\ $\mathrm{Yu} \mathrm{YaO}^{*} \quad$ Non-member
}

In this paper, a nonlinear positioning control scheme is proposed for a class of Euler-Lagrange systems acted by static friction. A compromise method of the constrained control input and the high accuracy position servo requirement is introduced to construct a $P D$-like controller. Based on the Filippov-framework, it's proved that the equilibrium point set of the closed-loop system is equal to the static solution set, which gives the maximum range of the state-steady servo error. By using the generalized Lyapunov method and Laselle's invariant principle, we prove that any trajectory of the closed-loop system will converge to the stationary set. Moveover, unlike the high gain feedback, the $P D$-like controller can regulate its feedback gain with respect to the servo error such that it is also available for the input constrained case. Finally, to demonstrate the proposed controller, some simulation results are presented.

Keywords: positioning control, Euler-Lagrange systems, nonsmooth control

\section{Introduction}

Positioning motion is a fundamental design issue for mechanical systems. The drawbacks caused by static friction, such as stick-slip limit-cycling, large steadystate errors, are still a challenging obstacle for positioning control in practice ${ }^{(1)}$. In the presence of such problems, a common approach to attain high accuracy positioning is to apply a high gain feedback, for example ${ }^{(2)-(4)}$. However, high gain control usually causes actuator saturation in implementation, especially for the case that large error occurs.

In order to trade off the design target and the capacity of the actuator, many feasible methods are proposed. Anti-windup control is an effective way to deal with the saturation of the actuator ${ }^{(5)}$. The controller can regulate its output into the linear region by means of a feedback loop from the actuator's status ${ }^{(6)}{ }^{(7)}$. Furthermore, low gain designs, such as ${ }^{(8)(9)}$, provide a gain turning matrix to adjust the feedback gain on-line. All these methods only focus on the nonlinearity of the actuator saturation or constrained control input and ignore the other nonlinearities of the systems which also influence the stability and the performance. Another mentionable obstacle for the high accuracy motion control is that the model of the friction force, especially static friction force, can not be exactly obtained. Many researches indicate that the static friction can be expressed by a discontinuous mapping with respect to the relative velocity

\footnotetext{
* K. Zheng and Y. Yao are with the Simulation and Control Center, Dept. of Control Science and Engineering, Harbin Institute of Technology, Harbin 150001, China

** T. Shen is with the Department of Mechanical Engineering, Sophia University, Tokyo
}

between the contact surface(10). However, the solution of a nonsmooth Euler-Lagrange equation, which will be used to establish the model of the mechanical systems acted by the static force, can not be analyzed by means of continuous differential equations.

In this paper, we will revisit the positioning control for a class of Euler-Lagrange systems which are driven by the saturated actuators. A discontinuous static mapping motivated by Southward et al. ${ }^{(10)}$, is used to represent the static friction force of the system. The common saturation function with respect to the control input is adopted to describe the bounded capacity of the actuators. Exactly speaking, with such two hard nonlinearities, the system becomes nonsmooth, i.e. such system must be modeled by a differential equation with discontinuous right-hand side. Filippov-framework ${ }^{(11)-(13)}$ is introduced to analyze behavior of the closed-loop system which has a nonempty stationary set. The result also provides the bounded of stationary set as well as an estimation of the static positioning error. Moveover, a nonlinear $P D$-like controller is proposed to avoid the actuator saturation and achieve the global stability of the stationary set which is proved to be the invariant set of the closed-loop system. It also shows that the feedback gain is scheduled with respect to the servo error and the positioning error can be rendered as small as any prespecified level. In order to demonstrate the proposed controller, a simulation study is carried out with a two-degree-of-freedom(2DOF) planner manipulator.

\section{Problem Formulation}

A standard method for modeling mechanical systems is via the so-called Euler-Lagrange equations (see ${ }^{(14)}$ )

$$
M(q) \ddot{q}+C(q, \dot{q}) \dot{q}+G(q)+\tau_{f}=\tau_{d} \ldots \ldots \ldots
$$


where

$q \quad$ Generalized coordinates $q \in \mathbb{R}^{n}$

$\dot{q} \quad$ Generalized velocities $\dot{q} \in \mathbb{R}^{n}$

$\ddot{q} \quad$ Generalized accelerations $\ddot{q} \in \mathbb{R}^{n}$

$M(q) \quad$ Inertia matrix $M(q) \in \mathbb{R}^{n \times n}$

$C(q, \dot{q}) \dot{q} \quad$ Coriolis force $C(q, \dot{q}) \in \mathbb{R}^{n \times n}$

$G(q) \quad$ Gravitational forces $G(q) \in \mathbb{R}^{n}$

$\tau_{f} \quad$ Static friction forces $\tau_{f} \in \mathbb{R}^{n}$

$\tau_{d} \quad$ Driving torque $\tau_{d} \in \mathbb{R}^{n}$

Moveover, dynamics given by (1) exhibits the following properties that are utilized in the subsequent control development and stability analysis.

(1) The inertia matrix $M(q)$ is positive definite, and bounded by the following inequality:

$$
m_{1} I \leq M(q) \leq m_{2} I \cdots \cdots \cdots \cdots \cdots \cdots(2)
$$

where $m_{1}$ and $m_{2}$ are positive constants.

(2) The inertia and the centripetal-Coriolis matrices satisfy the skew-symmetric relationship as follows

$$
\dot{M}(q)=C(q, \dot{q})+C^{T}(q, \dot{q})
$$

As proposed in ${ }^{(10)}$, the friction forces $\tau_{f}$, which is always acting on the opposite of the motion, is modeled by a set-valued function respect to the relative speed between the contract surface, that is

$$
\tau_{f}=D \mathcal{S g n}(\dot{q})
$$

where $D=\operatorname{diag}\left\{d_{i}\right\}, d_{i}>0, i=1, \ldots, n$ represents the bound of the friction force and $\mathcal{S g n}(\dot{\mathrm{q}})$ is a vector denoted by

$$
\operatorname{Sgn}(\dot{q})=\left[\operatorname{sgn}\left(\dot{q}_{1}\right) \operatorname{sgn}\left(\dot{q}_{1}\right) \ldots \operatorname{sgn}\left(\dot{q}_{n}\right)\right]^{T} \ldots \ldots
$$

The sign function $\operatorname{sgn}(\cdot)$ is defined as

$$
\operatorname{sgn}\left(\dot{q}_{i}\right)=\left\{\begin{array}{rl}
-1 & , \text { if } \dot{q}_{i}<0 \\
\xi_{i} & , \text { if } \dot{q}_{i}=0 \\
1 & , \text { if } \dot{q}_{i}>0
\end{array}, i=1,2, \ldots, n\right.
$$

where $\xi_{i} \in[-1,1], i=1,2, \ldots, n$ are unknown constants.

Consider the implementation of a real actuator, the driving torque $\tau_{d}$ is not infinite and constrained in a finite range. Such saturation nonlinearity is usually represented as follows

$$
\tau_{d_{i}}=\operatorname{sat}\left(\tau_{r_{i}}\right)=\left\{\begin{array}{cl}
B_{i}, & \text { if } \tau_{r_{i}}>B_{i} \\
\tau_{r_{i}}, & \text { if }\left|\tau_{r_{i}}\right| \leq B_{i}, i=1,2, \ldots, n \\
-B_{i}, & \text { if } \tau_{r_{i}}<-B_{i}
\end{array}\right.
$$

where $\tau_{r_{i}}, i=1, \ldots, n$, are torque level control for design and $B_{i}>d_{i}, i=1, \ldots, n$, denote the bound of the output for each freedom. Define $\mathcal{S}$ at $\left(\tau_{r}\right)=$ $\left[\operatorname{sat}\left(\tau_{r_{1}}\right) \operatorname{sat}\left(\tau_{r_{2}}\right) \ldots \operatorname{sat}\left(\tau_{r_{n}}\right)\right]^{T}$, equation (1) can be rewritten as

$$
M(q) \ddot{q}+C(q, \dot{q}) \dot{q}+G(q)+D \mathcal{S g n}(\dot{q})=\mathcal{S} \text { at }\left(\tau_{r}\right)
$$

Without loss of generality, if mechanism is centrosymmetric, the gravity force $G(q)$ is equal to zero by choosing proper coordinates in model (1). Use $q_{d}=$ $\left[\begin{array}{llll}q_{d_{1}} & q_{d_{2}} & \ldots & q_{d_{n}}\end{array}\right]^{T}$ to express the desired position of each freedom, and $x_{1}=\left[\begin{array}{llll}x_{1_{1}} & x_{1_{2}} & \ldots & x_{1_{n}}\end{array}\right]^{T}=$ $\left[\begin{array}{llll}\left(q_{1}-q_{d_{1}}\right) & \left(q_{2}-q_{d_{2}}\right) & \ldots & \left(q_{n}-q_{d_{n}}\right)\end{array}\right]^{T}$ to express the vector of the position servo error. Let $x_{2}=\dot{x}_{1}=$ $\left[\begin{array}{llll}x_{2_{1}} & x_{2_{2}} & \ldots & x_{2_{n}}\end{array}\right]^{T}$, the error system is represented as

$$
\left\{\begin{aligned}
\dot{x}_{1} & =x_{2} \\
M(q) \dot{x}_{2} & =-C(q, \dot{q}) x_{2}+\mathcal{S a t}\left(\tau_{r}\right)-D \mathcal{S} g n\left(x_{2}\right)
\end{aligned}\right.
$$

For above system, the positioning control design problem is to construct a proper driving torque $\tau_{r}$ to drive the positioning error as small as possible, i.e. we attempt to find a continuous feedback controller $\tau_{r}\left(x_{1}, x_{2}\right)$ such that for any initial condition $x_{1}\left(t_{0}\right), x_{2}\left(t_{0}\right)$, the closed-loop system is Lyapunov stable and $x_{1}(t)$ converges to a previously specified set $\Omega:=\left\{x_{1}|| x_{1_{i}} \mid \leq \varepsilon_{i}, i=1, \ldots, n\right\}$ and stays in this set as $t \rightarrow \infty$, where $\varepsilon_{i}, i=1, \ldots, n$ are arbitrarily small positive constants.

\section{Static Positioning Error of the Closed- loop System}

As mentioned in the introduction, the system of interest is a typical nonsmooth system. Due to the discontinuous right-hand side, the solution in Filippov sense satisfies the following differential inclusion

$$
\dot{x} \in K[f](x)
$$

where $x=\left[\begin{array}{ll}x_{1}^{T} & x_{2}^{T}\end{array}\right]^{T}$ is the state of the original equation $(9), K[f](x)$ is defined as the smallest convex closed set containing all the value of the vector function on the right-hand side of the equation. For system $(9), K[f](x)$ can be written as

$$
K[f](x)=K\left[\begin{array}{c}
x_{2} \\
-G(q) C(q, \dot{q}) x_{2}+G(q) \mathcal{S} \text { at }\left(\tau_{r}\right)-G(q) D \mathcal{S g n}\left(x_{2}\right)
\end{array}\right]
$$

where $G(q)=M^{-1}(q)$. Denote $G(q)=\left(g_{i j}(q)\right)_{n \times n}$ and $C(q, \dot{q})=\left(c_{i j}(q, \dot{q})\right)_{n \times n}$, briefly $\left(g_{i j}\right)$ and $\left(c_{i j}\right)$.

Moreover, the static solution is defined as any state $x$ which satisfies $0 \in K[f](x)$. As shown in the following lemma, the stationary set of the closed-loop system with any Lipschitz feedback controller may be a finite interval and the origin is not the unique static solution.

Lemma 1 For the closed-loop system of (9) with any static Lipschitz feedback controller $\tau_{r}\left(x_{1}, x_{2}\right)$ which satisfies $\tau_{r}=\mathcal{S}$ at $\left(\tau_{r}\right)$, i.e. $\left|\tau_{r_{i}}\right| \leq B_{i}, i=1, \ldots, n$, any $x \in \Omega$ is an equilibrium point of the system (10), where $\Omega=\left\{x \in \mathbb{R}^{2 n} \mid \tau_{r_{i}}\left(x_{1}, 0\right) \in d_{i} K[\operatorname{sgn}(0)], x_{2}=0, i=1, \ldots, n\right\}$

Proof: Obviously, all the discontinuous points belong to the set

$$
\mathcal{S}=\bigcup_{i=1}^{n} S_{i}
$$

where $S_{i}=\left\{x \in \mathbb{R}^{2 n} \mid x_{2_{i}}=0\right\}, i=1, \ldots, n$ are the switching surface of each freedom. It's obvious that $f(x)$ is continuous and $f(x) \neq 0$ for any $x \notin \mathcal{S}$. In other 
words, if the system has equilibrium point, it must be in $\mathcal{S}$. Hence, we focus on the set $\mathcal{S}$ only. Due to $S_{i} \subset \mathcal{S}, i=1, \ldots, n$, we can find all static solutions by searching the points $x$ satisfying $0 \in K[f](x)$ in each $S_{i}, i=1, \ldots, n$.

Note that, the whole space $\mathbb{R}^{2 n}$ is divided by the surface $S_{i}$ into the following two regions

$$
S_{i}^{+}=\left\{x \in \mathbb{R}^{2 n} \mid x_{2_{i}}>0\right\}, \quad S_{i}^{-}=\left\{x \in \mathbb{R}^{2 n} \mid x_{2_{i}}<0\right\}
$$

We can obtain the following limit values of the function $f(x)$ under approach to $S_{i}$ from $S_{i}^{+}$and $S_{i}^{-}$, respectively.

$$
\begin{aligned}
& f_{i}^{+}(x)=\left\{\begin{array}{c}
x_{2_{1}} \\
\cdots \\
x_{2_{n}} \\
\Sigma_{1}\left(x_{1}, x_{2}, \xi\right)+\sum_{j=1}^{n} g_{1 j} \tau_{r_{j}}\left(x_{1}, x_{2}\right)-g_{1 i} d_{i} \\
\ldots \\
\Sigma_{n}\left(x_{1}, x_{2}, \xi\right)+\sum_{j=1}^{n} g_{n j} \tau_{r_{j}}\left(x_{1}, x_{2}\right)-g_{n i} d_{i}
\end{array}\right. \\
& f_{i}^{-}(x)=\left\{\begin{array}{c}
x_{2_{1}} \\
\cdots \\
x_{2_{n}} \\
\Sigma_{1}\left(x_{1}, x_{2}, \xi\right)+\sum_{j=1}^{n} g_{1 j} \tau_{r_{j}}\left(x_{1}, x_{2}\right)+g_{1 i} d_{i} \\
\cdots \\
\Sigma_{n}\left(x_{1}, x_{2}, \xi\right)+\sum_{j=1}^{n} g_{n j} \tau_{r_{j}}\left(x_{1}, x_{2}\right)+g_{n i} d_{i}
\end{array}\right.
\end{aligned}
$$

where

$$
\Sigma_{p}\left(x_{1}, x_{2}, \xi\right)=-\sum_{\substack{m=1 \\ m \neq i}}^{n}\left(\sum_{j=1}^{n} g_{p j} c_{j m} x_{2_{m}}+d_{m} g_{p m} \xi_{m}\right)
$$

are briefly denoted by $\Sigma_{p}, p=1, \ldots, n$ and $\xi_{m} \in$ $K\left[\operatorname{sgn}\left(x_{2_{m}}\right)\right], m=1, \ldots, n, m \neq i$ are unknown constants. Since the normal vector of the switching sur-

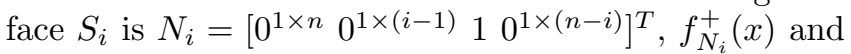
$f_{N_{i}}^{-}(x)$, which are the projections of the vectors $f_{i}^{+}(x)$ and $f_{i}^{-}(x)$ onto the normal to the surface $S_{i}$, can be calculated as follows

$$
\begin{array}{r}
f_{N_{i}}^{+}(x)=\Sigma_{i}\left(x_{1}, x_{2}, \xi\right)+\sum_{j=1}^{n} g_{i j} \tau_{r_{j}}\left(x_{1}, x_{2}\right)-g_{i i} d_{i} \\
\ldots \ldots \ldots \ldots \ldots \ldots \ldots \ldots \ldots \\
f_{N_{i}}^{-}(x)=\Sigma_{i}\left(x_{1}, x_{2}, \xi\right)+ \\
\sum_{j=1}^{n} g_{i j} \tau_{r_{j}}\left(x_{1}, x_{2}\right)+g_{i i} d_{i}
\end{array}
$$

Thus, from Lemma 3 in ${ }^{(11)}$, the differential inclusion (10) has a solution $x$ for $t \in\left[t_{0}, t_{1}\right]$ on the surface $S_{i}$, if and only if $x \in \Delta_{i}$ for all $t \in\left[t_{0}, t_{1}\right]$ where $\Delta_{i}$ is represented by

$$
\Delta_{i}=\left\{x \in S_{i} \mid f_{N_{i}}^{+} \leq 0, f_{N_{i}}^{-} \geq 0, f_{N_{i}}^{-}-f_{N_{i}}^{+}>0\right\}
$$

Furthermore, along the trajectories of the solution, $x$ satisfies

$$
\dot{x}=f_{i}^{\circ}(x)=\frac{f_{N_{i}}^{-}}{f_{N_{i}}^{-}-f_{N_{i}}^{+}} f_{i}^{+}(x)+\frac{f_{N_{i}}^{+}}{f_{N_{i}}^{-}-f_{N_{i}}^{+}} f_{i}^{-}(x)
$$

This leads the following conclusion: $x \in \Delta_{i}$ is an equilibrium in Filippov sense, if and only if $f_{i}^{\circ}(x)=0$ holds at the point $x$, i.e.

$$
0 \in f_{i}^{\circ}(x)=\left\{\begin{array}{c}
x_{1_{1}} \\
\ldots \\
x_{1_{(i-1)}} \\
0 \\
x_{1_{(i+1)}} \\
\ldots \\
x_{1_{n}} \\
\Sigma_{1}-\frac{g_{1 i}}{g_{i i}} \Sigma_{i}+\sum_{j=1}^{n} \frac{g_{1 j} g_{i i}-g_{1 i} g_{i j}}{g_{i i}} \tau_{r_{j}} \\
\ldots \\
\Sigma_{i-1}-\frac{g_{(i-1) i}}{g_{i i}} \Sigma_{i}+\sum_{j=1}^{n} \frac{g_{(i-1) j} g_{i i}-g_{(i-1) i} g_{i j}}{g_{i i}} \tau_{r_{j}} \\
\Sigma_{i+1}-\frac{g_{(i+1) i}}{g_{i i}} \Sigma_{i}+\sum_{j=1}^{n} \frac{g_{(i+1) j} g_{i i}-g_{(i+1) i} g_{i j}}{g_{i i}} \tau_{r_{j}} \\
\Sigma_{n}-\frac{g_{n i}}{g_{i i}} \Sigma_{i}+\sum_{j=1}^{n} \frac{g_{n j} g_{i i}-g_{n i} g_{i j}}{g_{i i}} \tau_{r_{j}}
\end{array}\right.
$$

Obviously, from this condition, we can get

$$
\left\{\begin{array}{l}
0=x_{2_{j}} \\
0 \in \tau_{r_{j}}\left(x_{1}, 0\right)+d_{j} \xi_{j}
\end{array}, \quad j=1, \ldots, n, j \neq i\right.
$$

Due to $\xi_{i} \in K\left[\operatorname{sgn}\left(x_{2_{i}}\right)\right]$, the above equations mean the range of $\tau_{r_{j}}\left(x_{1}, 0\right), j=1, \ldots, n, j \neq i$ is given by

$$
\tau_{r_{j}}\left(x_{1}, 0\right) \in K[\operatorname{sgn}(0)], j=1, \ldots, n, j \neq i \ldots
$$

Moveover, from the condition (19), we can obtain the range of $\tau_{r_{i}}$, that is

$$
\tau_{r_{i}}\left(x_{1}, 0\right) \in K[\operatorname{sgn}(0)]
$$

Hence, the static solution set on the switching surface $S_{i}$ is

$$
\Omega_{i}=\left\{x \in S_{i} \mid \tau_{r_{j}}\left(x_{1}, 0\right) \in K[\operatorname{sgn}(0)], j=1, \ldots, n, x_{2}=0\right\}
$$

Obviously, $\Omega_{i} \neq \phi, i=1, \ldots, n$. The equilibrium point set of the closed-loop is

$$
\begin{array}{r}
\Omega=\bigcup_{i=1}^{n} \Omega_{i}=\left\{x \in \mathbb{R}^{2 n} \mid \tau_{r_{j}}\left(x_{1}, 0\right) \in K[\operatorname{sgn}(0)],\right. \\
\left.x_{2}=0, j=1, \ldots, n\right\}
\end{array}
$$

Lemma 1 shows the equilibrium of system (9) with a static feedback controller is not unique. Obviously, if there are not any actuator saturations, it is a possible case that the system converges to a point $x \in \Omega$ where $x_{1_{i}} \neq 0, i=1, \ldots, n$. More information of the stationary set is that the set has a definite bound. In this case, 
Lemma 1 may provide an estimation for the bound of static positioning error.

Actually, when we focus on the static solution at one switching surface, the existence condition of the static solution requires the constraint (22) for other freedom. Hence, $\Omega_{1}=\cdots=\Omega_{n}$. This is a common region of the solution on each switching surface. Moreover, it should be noted that the physical meaning of the static set is zero velocity of the motion parts. This fact implies that the trajectory will stay in the set.

The static solution also gives us a hint on the error range. If we want to enhance the servo accuracy, a feasible way is to drive the trajectory into the static solution set whose range can be regulated by the controller. In next section, the design problem is discussed.

\section{Nonlinear Constrained Control design}

As we all know, the fixed gain feedback, such as $P D$ control, usually causes large control input as the initial state is far from the equilibria. It's also an important reason for the actuator saturation. In this section, we will give a nonlinear $P D$-like controller to avoid the actuator saturation. Moreover, the accuracy requirement can be also guaranteed by the controller.

It is noticeable that the saturation function is a bounded function respect to the input. If we design a continuous state feedback law who has the bound of saturation, the actuator saturation can be avoided. Define the $P D$-like controller as

$$
\tau_{r}=-K_{p} \mathcal{A} \operatorname{rctg}\left(H x_{1}\right)-K_{d} \mathcal{A} \operatorname{rctg}\left(x_{2}\right) \cdots \cdots
$$

where $K_{p}=\operatorname{diag}\left\{k_{p_{i}}\right\}, K_{d}=\operatorname{diag}\left\{k_{d_{i}}\right\}$ and $H=$ $\operatorname{diag}\left\{h_{i}\right\}$ with $k_{p_{i}}>0, k_{d_{i}}>0, h_{i}>0, i=1, \ldots, n$. $\mathcal{A r c t g}\left(H x_{1}\right)$ and $\operatorname{Arctg}\left(x_{2}\right)$ are vectors and defined as

$$
\operatorname{Arctg}\left(H x_{1}\right)=\left[\arctan \left(h_{1} x_{1_{1}}\right) \ldots \arctan \left(h_{1} x_{1_{n}}\right)\right]^{T}
$$

and

$$
\operatorname{Arctg}\left(x_{2}\right)=\left[\arctan \left(x_{2_{1}}\right) \ldots \arctan \left(x_{2_{n}}\right)\right]^{T} \ldots
$$

The function $\arctan (x)$ is a monotone continuous odd function with bounded value, that is

(1) $\arctan (x) \in\left[-\frac{\pi}{2}, \frac{\pi}{2}\right]$

(2) $\arctan (-x)=-\arctan (x)$

Due to the monotonicity of arctangent function, the result of Lemma 1 can be easily used to derive the static solution of the system with a $P D$-like controller (27). This result is shown in the following theorem.

Theorem 1 For the closed-loop system of (9) with the $P D$-like controller (27), if the design parameters $K_{p}$ and $K_{d}$ satisfy

$$
k_{p_{i}}+k_{d_{i}} \leq \frac{2}{\pi} B_{i}, i=1
$$

then any $x=\left[\begin{array}{ll}x_{1}^{T} & x_{2}^{T}\end{array}\right]^{T} \in \Omega$ is an equilibrium of the closed-loop system, where

$\Omega=\left\{x \in \mathbb{R}^{2 n}|| x_{1_{j}} \mid \leq \tan \left(\frac{d_{j}}{k_{p_{j}}}\right) / h_{j}, x_{2}=0, j=1, \ldots, n\right\}$

$$
\ldots \ldots \ldots \ldots \ldots \ldots \ldots \ldots \ldots+(31)
$$
that

$$
\begin{aligned}
\tau_{r_{i}} & =-k_{p_{i}} \arctan \left(h_{i} x_{1_{i}}\right)-k_{d_{i}} \arctan \left(x_{2_{i}}\right) \\
& \leq k_{p_{i}}\left|\arctan \left(h_{i} x_{1_{i}}\right)\right|+k_{d_{i}}\left|\arctan \left(x_{2_{i}}\right)\right| \ldots \\
& \leq\left(k_{p_{i}}+k_{d_{i}}\right) \frac{\pi}{2} \leq B_{i}, \quad i=1, \ldots, n
\end{aligned}
$$

It implies that the controller (27) with (30) satisfies the condition of Lemma 1. Based on Lemma 1, any static solution should satisfy

$$
-k_{p_{i}} \arctan \left(h_{i} x_{1_{i}}\right) \in d_{i} K[\operatorname{sgn}(0)], i=1, \ldots, n
$$

and $x_{2}=0$. An equivalent result is

$$
\left|x_{1_{i}}\right| \leq \tan \left(\frac{d_{i}}{k_{p_{i}}}\right) / h_{i}, i=1, \ldots, n \ldots \ldots \ldots
$$

Then the theorem follows.

Theorem 1 implies that the bound of the positioning error is related to the design parameter $H$ and $K_{p}$. Therefore, if the closed-loop system is stable, the proposed controller (27) is a feasible controller of our design problem. The following theorem indicates that if the parameters satisfy the condition given in the theorem, the equilibria set achieves the global stability in Lyapunov sense.

Theorem 2 Consider the system (9) with the $P D$ like controller (27). For any prespecified positive value $\varepsilon_{i}, i=1, \ldots, n$ and any initial condition, if $H, K_{p}, K_{d}$ satisfy

(1) $k_{p_{i}}+k_{d_{i}} \leq \frac{2}{\pi} B_{i}, i=1, \ldots, n$

(2) $k_{p_{i}} \leq 2 d_{i}, i=1, \ldots, n$

(3) $\frac{1}{h_{i}} \tan \left(\frac{d_{i}}{k_{p_{i}}}\right)<\varepsilon_{i}, i=1, \ldots, n$

then the closed loop is globally stable and $\lim _{t \rightarrow \infty}\left|x_{1_{i}}\right|=c_{i}<$ $\varepsilon_{i}, i=1, \ldots, n$.

Proof: As proved in the Theorem 1, condition 1 guarantees that the output of the $P D$-like controller is in the linear region of the saturation, i.e. $\mathcal{S}$ at $\left(\tau_{r}\right)=\tau_{r}$.

An extended Lyapunov method for nonsmooth system is introduced here to prove the stability of the closedloop system in Filippov sense. Unlike usual quadratic Lyapunov function, we introduce a non-quadratic Lyapunov function candidate to verify the attractivity. Define the Lyapunov function candidate

$$
V\left(x_{1}, x_{2}\right)=x_{1}^{T} K_{p} \operatorname{Arctg}\left(H_{p} x_{1}\right)+\frac{1}{2} x_{2}^{T} M\left(x_{1}\right) x_{2}
$$

Due to the odd property of arctan function, $V\left(x_{1}, x_{2}\right)$ is a positive function. $\mathrm{By}{ }^{(12)}$, we should calculate the generalized time derivative of $V$ which is defined as

$$
\dot{\tilde{V}}=\bigcap_{\zeta \in \partial V} \zeta^{T} \cdot K[f](x) \cdot
$$

where $\partial V$ is the Clarke's generalized gradient ${ }^{(12)}$. In fact, $\dot{\tilde{V}}$ is defined as a set. Let $\left.\dot{\tilde{V}}\right|_{x \in D}$ denote the set of the element in $\dot{\tilde{V}}$ when $x$ is in a region $D$. In this case, since $V\left(x_{1}, x_{2}\right)$ is a continuous function respect to the state $x_{1}$ and $x_{2}, \partial V$ is equal to the normal gradient 
$\partial V / \partial x$. Furthermore, it is clear that $K[f](x)$ involves single element calculated by the right-hand side with a definite value of $\mathcal{S}_{\text {gn }}\left(x_{2}\right)$ whenever $x \notin \mathcal{S}$, i.e. in such region, we have

$$
\begin{aligned}
\left.\dot{\tilde{V}}\right|_{x \notin \mathcal{S}}= & \sum_{j=1}^{n}\left(k_{p_{j}} \frac{h_{j} x_{2_{j}} x_{1_{j}}}{1+h_{j}^{2} x_{1_{j}}^{2}}-d_{j}\left|x_{2_{j}}\right|\right)-x_{2}^{T} K_{d} \mathcal{A} \operatorname{rctg}\left(x_{2}\right) \\
& +\frac{1}{2} x_{2}^{T}(\dot{M}-2 C) x_{2} \ldots \ldots \ldots \ldots \ldots \ldots
\end{aligned}
$$

By applying the skew-symmetric property (3) of Lagrange systems,

$$
\left.\dot{\tilde{V}}\right|_{x \notin \mathcal{S}} \leq \sum_{j=1}^{n}\left(\frac{1}{2} K_{p_{j}}-d_{j}\right)\left|x_{2_{j}}\right|-x_{2}^{T} K_{d} \operatorname{Arctg}\left(x_{2}\right)
$$

Because $x_{2}^{T} K_{d} \mathcal{A} \operatorname{rctan}\left(x_{2}\right) \geq 0$. With condition 2, we can get $\left.\dot{\tilde{V}}_{1}\right|_{x \notin \mathcal{S}} \leq 0$.

Moreover, on any switching surface $S_{i}, i=1, \ldots, n$, $K[f](x)$ is defined as a convex closure represented by

$$
f_{i}^{\circ}(x) \in K[f](x)=\lambda_{i} f_{i}^{+}(x)+\left(1-\lambda_{i}\right) f_{i}^{-}(x) \cdots
$$

where $\lambda_{i} \in[0,1]$ is a constant, $f_{i}^{+}(x)$ and $f_{i}^{-}(x)$ are given in (15) and (16), respectively. Hence, the generalized time derivative of $V_{0}$ can be calculated by

$$
\begin{array}{r}
\left.\dot{\tilde{V}}_{0}\right|_{x \in S_{i}}=\sum_{\substack{j=1 \\
j \neq i}}^{n}\left(\frac{1}{2} K_{p_{j}}-d_{j}\right)\left|x_{2_{j}}\right|-x_{2}^{T} K_{d} \operatorname{Arctan}\left(x_{2}\right) \\
\ldots \ldots \ldots \ldots \ldots \ldots \ldots
\end{array}
$$

For the whole state space, since the value of any element in $\dot{\tilde{V}}$ is not greater than zero, we can conclude that there exists $D \subset \mathbb{R}^{2 n}$ such that $\eta \leq 0$ for any $\left.\eta \in \dot{\tilde{V}}_{0}\right|_{\forall x \in D}$ and $\eta=0$ iff $x \in S_{1} \cap \cdots \cap S_{n}$ which is a subset of $D$. Based on the extended LaSalle's invariant set principle ${ }^{(12)}$, any trajectory starting in $D$ will approach the largest invariant set in the set $S_{1} \cap \cdots \cap S_{n}$. Obviously, the largest invariant set is equal to the solution set in which any solution satisfies

$$
\dot{x}=f_{1}^{\circ}(x)=\cdots=f_{n}^{\circ}(x) \cdots
$$

where $f_{i}^{\circ}(x), i=1, \ldots, n$ are defined in (39). It can be obtained that the solution is equal to the equilibrium point set $\Omega$ in Theorem 1 .

From Theorem 1, the trajectories will approach its static solution which is bounded by $\frac{1}{h_{i}} \tan \left(\frac{d_{i}}{k_{p_{i}}}\right), i=$ $1, \ldots, n$. With the condition 3 , the static solution is bounded by $\varepsilon_{i}, i=1, \ldots, n$, that is

$$
\left|x_{1_{i}}\right|=c_{i}<\frac{1}{h_{i}} \tan \left(\frac{d_{i}}{k_{p_{i}}}\right)<\varepsilon_{i}, i=1, \ldots, n \ldots
$$

Remark 1 There are three conditions for choosing the parameter in Theorem 2. These three conditions take different design targets. The condition 1 as well as the condition of Theorem 1 guarantees that the controller works in the linear region of the actuator. For the condition 2 , it is for the stability of the closed-loop system. The condition 3 is introduced for the accuracy requirement.
It's obvious that for such a nonlinear $P D$-like controller, the usage of proportional parameter $K_{p}$ is not like the traditional $P D$ controller. The value of $K_{p}$ is not determined by the accuracy requirement but by the condition 1 and 2 of Theorem 2. Of course, the accuracy requirement is ensured by regulating $H$. In the view of reducing the positioning error, $H$ can be considered as the feedback gain. We also find that the choice of $H$ has not any influence on the stability of the closed-loop system. Therefore, $H$ can be chosen separately with other parameters.

\section{Numerical Example}

In order to illustrate the effect of the proposed $P D$ like controller, a numerical example is carried out. A 2DOF planner manipulator is examined as the plant. The parameters are represented as

$$
\begin{aligned}
& M(q)=\left[\begin{array}{cc}
J_{1}+2 R \cos q_{1} & J_{2}+R \cos q_{2} \\
J_{2}+R \cos q_{2} & J_{2}
\end{array}\right] \ldots \\
& C(q, \dot{q})=\left[\begin{array}{cc}
-2 R \dot{q}_{2} \cos q_{2} & -R \dot{q}_{2} \cos q_{2} \\
R \dot{q}_{2} \cos q_{2} & 0
\end{array}\right] \ldots \\
& \tau_{f}=\left[\begin{array}{c}
d_{1} \operatorname{sgn}\left(\dot{q}_{1}\right) \\
d_{2} \operatorname{sgn}\left(\dot{q}_{2}\right)
\end{array}\right] \ldots \ldots \ldots \ldots \ldots \ldots
\end{aligned}
$$

where $J_{1}=0.3, J_{2}=0.03, R=0.031, d_{1}=1.5, d_{2}=1$. For the saturation bound, $B_{1}=B_{2}=5$. Let the accuracy for the two freedoms to be 0.01 , respectively. According to Theorem 2 , the design parameters $k_{p_{i}}, k_{d_{i}}$, $h_{i}, i=1,2$, should satisfy the following inequalities

$$
\left\{\begin{array}{l}
k_{p_{1}}+k_{d_{1}} \leq \frac{10}{\pi}, k_{p_{2}}+k_{d_{2}} \leq \frac{10}{\pi} \\
k_{p_{1}} \leq 3, k_{p_{2}} \leq 2 \\
\frac{1}{h_{1}} \tan \left(\frac{1.5}{k_{p_{1}}}\right)<0.01, \frac{1}{h_{2}} \tan \left(\frac{1}{k_{p_{2}}}\right)<0.01
\end{array}\right.
$$

In this case, we choose the parameters as

$$
k_{p_{1}}=k_{p_{2}}=\frac{6}{\pi}, k_{d_{1}}=k_{d_{2}}=\frac{4}{\pi}, h_{1}=100, h_{2}=60
$$

To show the effectiveness of our design, the initial conditions of the simulation are set to be $\left(q_{1}, \dot{q}_{1}\right)=$ $(10,-10),\left(q_{2}, \dot{q}_{2}\right)=(10,-10)$. The phase loci of the response are drawn in Fig. 1. The control inputs are plotted in Fig. 2.

Although the positioning errors of two degrees of freedom do not converge to the origin, they satisfy the accuracy requirement. Obviously, the driving torque is also in the linear region of the saturation although the initial condition has a large variation. Actually, the outputs of controller will be restricted in the linear region for any initial condition, as long as the parameters satisfy the conditions of Theorem 2. In other words, we can use arbitrary initial condition for the system to verify our results. Another remarkable point is that the response speed of the motion system is not concerned in our design, though it's also an important design target. Generally speaking, in order to improve the response speed, we may assign the value of the parameters $K_{p}$ and $K_{d}$ as large as possible in the implementation. 

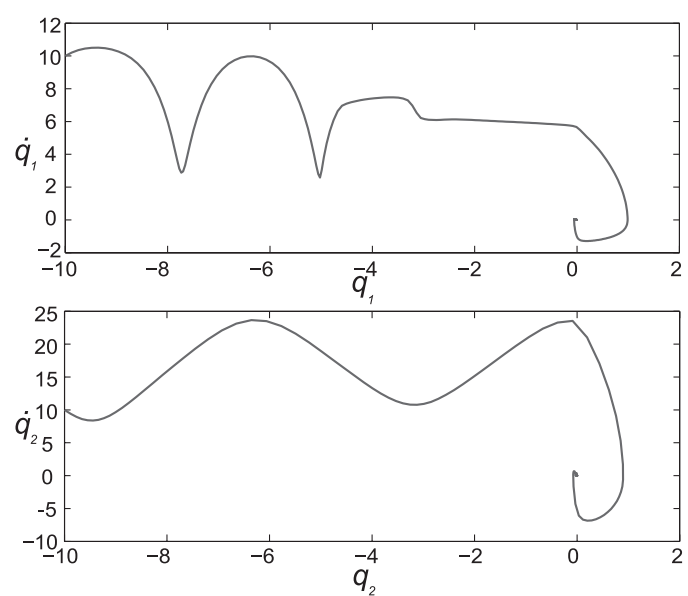

Fig. 1. Response phase locus of each freedom.
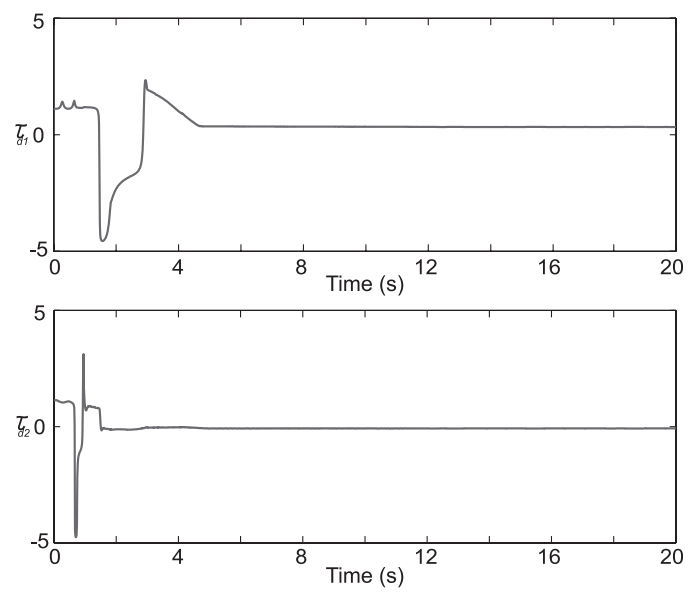

Fig. 2. Driving torque of each freedom.

\section{Conclusion}

In this paper, we present an application of the nonsmooth control theory in positioning control of a class of Euler-Lagrange systems. Due to the static friction, the equilibrium of the system with any Lipschitz continuous controller is not unique but multiple in Filippov sense. In order to overcome the input saturation, a nonlinear $P D$-like controller is proposed. The stability and the accuracy requirement is separately guaranteed by the different design parameters of the controller. We also prove that the stationary set is the largest invariant set of the closed-loop system. With the feasible control parameters, the positioning error can be restricted to any given level. Simulation results also show that this design procedure gives more freedoms of controller than usual fixed gain control.

(Manuscript received May 29, 2007, revised Oct. 1, 2007)

\section{References}

(1) N. V. D. Wouw and R. I. Leine: "Attractivity of equilibrium sets of systems with dry friction", Nonlinear Dynamics, Vol.35, No.1, pp.19-39 (2004)

(2) N. Koreta, H. Okitomo, K. Tsumura, K. Takeuchi, and T. Egawa: "Study of high accuracy of machine tool with bangbang control", Journal of Japan Society for Precision Engineering, Vol.60, No.3, pp.427-431 (1994)
( 3 ) X. Yue, D. M. Vilathgamuwa, and K.-J. Tseng: "Robust adaptive control of a three-axis motion simulator with state observers", IEEE/ASME Transaction on Mechatronics, Vol.10, No.4, pp.437-448 (2005)

(4) S. S. Ge, T. H. Lee, and S. X. Ren: "Adaptive friction compensation of servo mechanisms", International Journal of Systems Science, Vol.32, No.4, pp.523-532 (2001)

( 5 ) Y. Peng, D. Vrancic, and R. Hanus: "Anti-windup, bumpless, and conditioned transfer techniques for PID controllers", IEEE Control Systems Magazine, Vol.16, No.4, pp.48-57 (1996)

(6) J. S. Shamma: "Anti-windup via constrained regulation with observers", in Proceedings of the American Control Conference, San Diego, California (1999)

( 7 ) M. M. Mayuresh V. Kothare, Peter J. Campo: "A unified framework for the study of anti-windup designs", Automatica, Vol.30, No.2, pp.1869-1883 (1994)

(8) A. Saberi, Z. Lin, and A. R. Teel: "Control of linear systems with saturating actuators", IEEE Transactions on Automatic Control, Vol.41, No.3, pp.368-378 (1996)

(9) T. $\mathrm{Hu}$ and $\mathrm{Z}$. Lin: "On improving the performance with bounded continuous feedback laws", IEEE Transactions on Automatic Control, Vol.47, No.9, pp.1570-1575 (2002)

(10) S. S. C., R. C. J., and M. C.R.: "Robust nonlinear stick-slip friction compensation", ASME Journal of Dynamic Systems, Measurement and Control, Vol.113, No.6, pp.639-645 (1991)

(11) A. Filippov: "Differential equations with discontinous righthand side", American Mathematical Society Translations, Vol.42, No.2, pp.354-362, (1964)

(12) D. Shevitz and B. Paden: "Lyapunov stability theory of nonsmooth systems", IEEE Transactions on Automatic Control, Vol.39, No.9, pp.1910-1914 (1994)

(13) J. Alvarez, I. Orlov, and L. Acho: "An invariance principle for discontinuous dynamic systems with application to a coulomb friction oscillator", Journal of Dynamic Systems, Measurement, and Control, Vol.122, No.4, pp.687-690 (2000)

(14) R. Ortega: Passivity-Based Control of Euler-Lagrange Systems: Mechanical, Electrical and Electromechanical Applications, Springer Verlag (1998)

Kai Zheng (Non-member) received the B.E. and M.E. de-

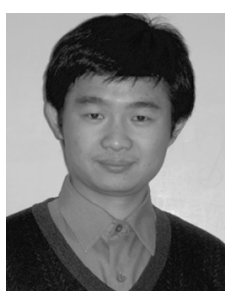
grees in Control Engineering from Harbin Institute of Technology, China, in 2002 and 2004, respectively. Now, he is a Ph.D. candidate with the Control and Simulation Center, Harbin Institute of Technology. He is also a collaborator of the Department of Mechanical Engineering, Sophia University. His current research interests include nonlinear dynamic systems and automotive control.

Tielong Shen (Member) received the Ph.D. degree in Me-

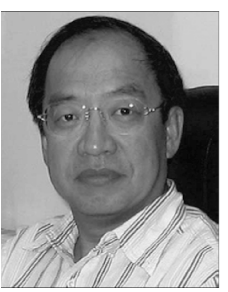
chanical Engineering from Sophia University, Tokyo, Japan, 1992. He is now serving as Associate Professor in Sophia University. His research interests include robust control theory, nonlinear and adaptive control, and recently in mechanical systems, power systems, and automatic systems.

Yu Yao (Non-member) received the Ph.D. degree in Control

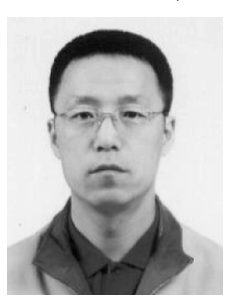
Engineering from Harbin Institute of Technology, China, 1990. Now he is a professor in Control Engineering and the Dean of School of Astronautics, Harbin Institute of Technology. His research interests include nonlinear system, hybrid systems design and guidance for flight control systems and simulation of aircraft. 